\title{
Poly-I:C Decreases Dendritic Cell Viability Independent of PKR Activation
}

\author{
Hjalte List Larsen, Anders Elm Pedersen \\ Department of International Health, Immunology and Microbiology, The Panum Institute, \\ University of Copenhagen, Copenhagen, Denmark \\ Email: hjaltel@sund.ku.dk, andersep@sund.ku.dk
}

Received March 1, 2012; revised April 2, 2012; accepted April 12, 2012

\begin{abstract}
Vaccination with tumor-antigen pulsed, monocyte-derived dendritic cells (DCs) has emerged as a promising strategy in cancer immunotherapy. The standard DC maturation cocktail consists of a combination of tumor necrosis factor- $\alpha$ (TNF- $\alpha$ )/interleukin (IL)-1 $\beta / \mathrm{IL}-6$ and prostaglandin $\mathrm{E}_{2}\left(\mathrm{PGE}_{2}\right)$ for generation of standard DCs (sDCs). In order to improve IL-12p70 production and cytotoxic T-lymphocyte (CTL) induction, a novel cocktail composed of TNF- $\alpha / \mathrm{IL}-1 \beta /$ interferon (IFN)- $\alpha / \mathrm{IFN}-\gamma$ and polyinosinic:polycytidylic acid (Poly-I:C) has been introduced to generate so-called $\alpha$-Type-1 polarized DCs $(\alpha \mathrm{DC} 1 \mathrm{~s})$. We and others have previously performed a comprehensive comparison of sDCs and $\alpha \mathrm{DC} 1 \mathrm{~s}$. Here we demonstrate that the viability of $\alpha \mathrm{DC} 1 \mathrm{~s}$ is lowered compared to sDCs and that DC apoptosis is mediated by Poly-I:C. We speculated that activation of protein kinase R (PKR) could mediate the observed apoptosis, but despite significantly higher PKR expression in $\alpha \mathrm{DC} 1 \mathrm{~s}$ compared to sDCs and induction of active threonine (Thr) 446 autophosphorylation of PKR in $\alpha$ DC1s, Poly-I:C did not influence total PKR expression or autophosporylation, indicating PKR-independent Poly-I:C-induced DC apoptosis.
\end{abstract}

Keywords: Cancer Immunotherapy; Dendritic Cells; Poly-I:C; PKR

\section{Introduction}

In therapeutic cancer vaccination with monocyte-derived DCs [1], maturation is mandatory for proper T-cell stimulation [2]. DCs maturated according to the standard cytokine cocktail consisting of TNF- $\alpha /$ IL- $1 \beta /$ IL- 6 and $\mathrm{PGE}_{2}$ [3] show high expression of maturation markers CD80, CD83, CD86, CD134L and CCR7, but low secretion of IL-12p70 [4] which may have negative consequences for activation of appropriate $\mathrm{T}$ cell responses such as TH1 cells and cytotoxic T lymphocytes (CTLs). In the search of cytokine cocktails that increase DC IL-12p70 secretion, DCs maturated according to the $\alpha$-Type-1 polarizing cocktail containing TNF- $\alpha /$ IL-1 $\beta /$ Poly-I:C/IFN- $\alpha$ and IFN- $\gamma$ were developed, as these DCs produce large amounts of IL-12p70 [4,5]. We and others have characterized cytokine secretion and capacity for $\mathrm{T}$ cell activation in details elsewhere $[4,5]$ and it has been found that $\alpha \mathrm{DC} 1 \mathrm{~s}$ may have a reduced capacity for $\mathrm{T}$ cell activation under certain conditions [4]. In addition to the criterias for successful DC generation investigated elsewhere, viability is an important factor. Here, we report a decreased viability of $\alpha \mathrm{DC} 1 \mathrm{~s}$ and investigate a potential role of PKR mediated mechanisms.

The double-stranded RNA (dsRNA)-analog Poly-I:C included in the $\alpha$-Type-1 polarizing cocktail is a potential inducer of apoptosis, as cytosolic Poly-I:C is capable of activating the dsRNA-activated protein kinase (PKR) [6]. Upon dsRNA/Poly-I:C binding, PKR is autophosphorylated on multiple sites including Thr446 and Thr451 [7], enabling signal transduction which can induce global translation inhibition and apoptosis [8]. Furthermore, Poly-I:C can be detected by toll-like receptor 3 (TLR3) in the endosomal compartment of DCs [9] and it has been reported that PKR is recruited to a large signaling complex following Poly-I:C binding to TLR3 [10]. We thus speculated that Poly-I:C is the cause of lowered $\alpha \mathrm{DC} 1$ viability as compared to sDCs, and that such a PolyI:C-mediated induction of apoptosis was mediated by PKR activation. In this case, siRNA mediated silencing of PKR-dependent apoptotic pathways could be a future strategy for improved efficacy of therapeutic $\alpha \mathrm{DC} 1 \mathrm{~s}$.

Here, we report that absence of Poly-I:C from the $\alpha$-Type-1 polarizing cocktail significantly improved $\alpha \mathrm{DC} 1$ viability to levels comparable to sDCs without significantly decreasing CD80, CD83 and CD86 expression. $\alpha \mathrm{DC} 1 \mathrm{~s}$ expressed significantly higher amounts of PKR than iDCs and sDCs, with PKR expression being dependent on the presence of IFNs in the maturation cocktail but not on Poly-I:C. We were also unable to detect an 
effect of Poly-I:C on PKR autophosphorylation in mature DCs, suggesting that the observed lowered viability of $\alpha \mathrm{DC} 1 \mathrm{~s}$ was PKR-independent.

\section{Methods}

\subsection{Generation of Human Monocyte-Derived DCs}

Human monocyte-derived DCs were generated as previously described with GM-CSF + IL-4 [11] from buffy coats after informed consent from healthy donors at the local department of clinical immunology. Maturation of iDCs was performed on day 7. sDCs were maturated with TNF- $\alpha(10 \mathrm{ng} / \mathrm{mL}), \mathrm{IL}-1 \beta(10 \mathrm{ng} / \mathrm{mL})$, IL-6 (10 $\mathrm{ng} / \mathrm{mL}$ ) (all cytokines from Peprotech) and $1 \mu \mathrm{g} / \mathrm{mL}$ $\mathrm{PGE}_{2}$ (Prostin E2, Pfizer). The $\alpha \mathrm{DC} 1$ phenotype was induced by TNF- $\alpha(10 \mathrm{ng} / \mathrm{mL}), \mathrm{IL}-1 \beta(10 \mathrm{ng} / \mathrm{mL})$, IFN- $\alpha$ $(10 \mathrm{ng} / \mathrm{mL})$, IFN- $\gamma(10 \mathrm{ng} / \mathrm{mL})$ (all cytokines from Peprotech) and $20 \mu \mathrm{g} / \mathrm{mL}$ Poly-I:C (Sigma Aldrich). DCs were maturated 48 hours prior to analysis.

\subsection{Flow Cytometry}

Flow cytometry was performed using antibodies against the following surface proteins: CD11c, CD80, CD83 and CD86 (BD-Pharmingen). Isotope control antibodies were obtained from DAKO. Detection of apoptotic DCs were performed using annexin V-FITC apoptosis detection Kit I (BD Pharmingen) according to the manufacturer's protocol. Data acquisition and analysis was performed on a FACSCalibur flow cytometer (BD) using CellQuest software (BD Biosciences). DCs were identified as the large granular cell population based on forward scatter (FSC)/side scatter (SSC) linear dot plots.

\subsection{Western Blotting}

DCs were lysed in RIPA buffer supplemented with proteinase inhibitor cocktail (Sigma Aldrich), PMSF, NaF and $\mathrm{Na}_{3} \mathrm{VO}_{4}$ during 20 minutes of centrifugation at $14,000 \mathrm{~g}, 4^{\circ} \mathrm{C}$. Lysates were treated with SDS and heated for 5 minutes prior to loading onto $4 \%-12 \%$ Bis-Tris gels (Invitrogen). Electrophoresis was performed in MES running buffer (Invitrogen) for 1 hour at $200 \mathrm{~V}$. Blotting was performed at $30 \mathrm{~V}$ for 1 hour onto nitrocellulose membranes (Invitrogen) in transfer buffer (Invitrogen). Membranes were blocked and probed with polyclonal goat anti-pPKR (Thr446) antibody, polyclonal rabbit anti-PKR antibody (both Santa Cruz Biotechnology, Inc.) or goat anti-GAPDH antibody (AbCam) over night at $4{ }^{\circ} \mathrm{C}$. Secondary horse radish peroxidase (HRP)-conjugated polyclonal antibodies (DAKO) were added and the membrane was incubated 1 hour at room temperature. The membrane was washed and visualized using an enhanced chemiluminescence detection system (ECL plus
Western Blotting Detection system, GE Healthcare) according to the manufacturer's instructions. Detection of HRP-generated precipitates was performed on a Typhoon Scanner 9410 with Typhoon Scanner software (both from Amersham Biosciences). The same software was used to analyze band intensity, with PKR and pPKR intensity being normalized to GAPDH expression.

\section{Results}

\subsection{Phenotype after Maturation}

Day 7 IL-4 and GM-CSF differentiated monocyte-derived DCs were identified as large granular cells expressing the DC marker CD11c (Figures 1(A) and (B)). Maturation using the two established cocktails for generation of sDCs and $\alpha \mathrm{DC} 1 \mathrm{~s}$ both significantly increased the expression of CD80 (p $<0.05$ and $p<0.01$ respectively) and $\operatorname{CD} 83$ ( $p<0.05$ and $p<0.05$ respectively) compared to iDCs, while a stable high expression level of CD86 was maintained from iDCs into mature DCs. CD83 expression in $\mathrm{sDC}$ was less pronounced in these DCs from healthy donors as compared to our previous results from cancer patient derived DCs [4]. Removal of individual cytokine components from the $\alpha$-Type-1 polarizing cocktail did not induce any significant changes in the expression of the three maturation markers, however absence of Poly-I:C or IFN- $\alpha$ reduced the expression of both CD80 and CD83 compared to expression levels in $\alpha \mathrm{DC} 1 \mathrm{~s}$. These observed reductions in CD80 and CD83 expression were not statistical significant but consistent in all donors. It was previously published that IL-12p70 secretion was dependent on IFN- $\gamma$ or Poly-I:C with the largest contribution from IFN- $\gamma$ [5]. For T cell activation, $\alpha \mathrm{DC} 1 \mathrm{~s}$ have been shown to be superior for CTL activation [5], whereas assays mostly reflecting $\mathrm{CD} 4+\mathrm{T}$ cell responses has demonstrated a decreased capacity for T cell activation as compared to SDCs [4].

\subsection{Viability of ex Vivo Generated DCs}

Annexin V-FITC and propidium iodide (PI) staining revealed a tendency toward that sDCs were more viable (mean of all donors: $79.4 \%$ living cells) as compared to $\alpha \mathrm{DC} 1 \mathrm{~s}$ (mean of all donors: $71.7 \%$ living cells), a difference that was not statistical significant $(p=0.069)$ but consistent in all donors. Data from one donor is shown in Figure 2(A) and mean of all donors in Figure 2(B). In order to identify the cause of the viability changes in $\alpha \mathrm{DC} 1 \mathrm{~s}$, all single components from the cocktail was systematically excluded (Figure 2(B)). Exclusion of Poly-I:C from the $\alpha$-type-1 polarizing cocktail significantly improved the viability of DCs from a mean of $71.7 \%$ $(\alpha \mathrm{DC} 1 \mathrm{~s})$ to $79.9 \%$ living cells $(\mathrm{p}=0.02)$, thus increasing $\mathrm{DC}$ viability to a level comparable to sDCs. The viability 


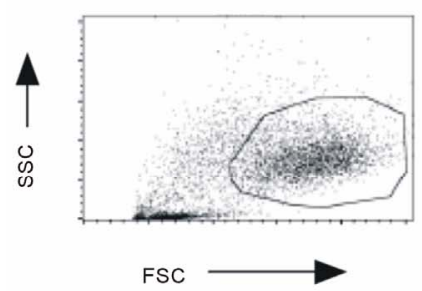

(A)

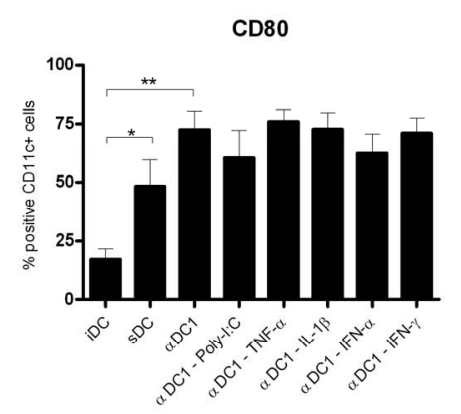

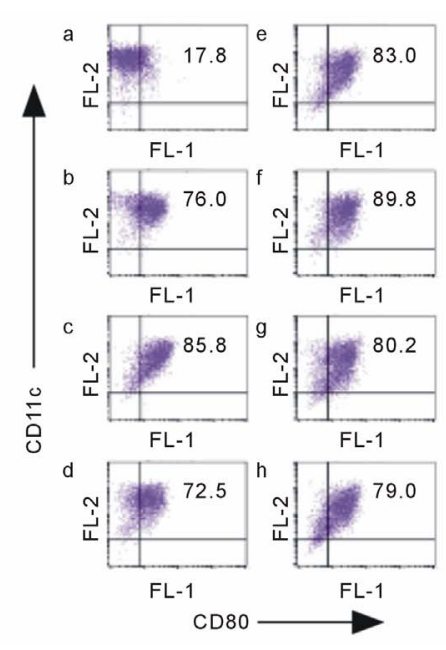

CD83

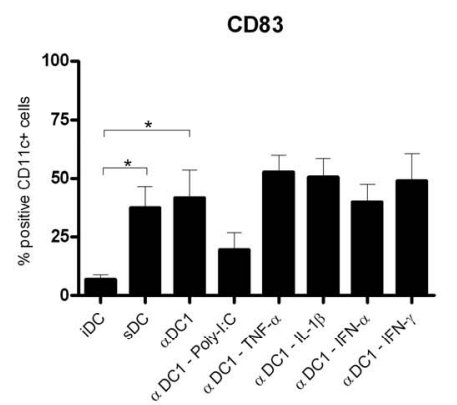

(C)
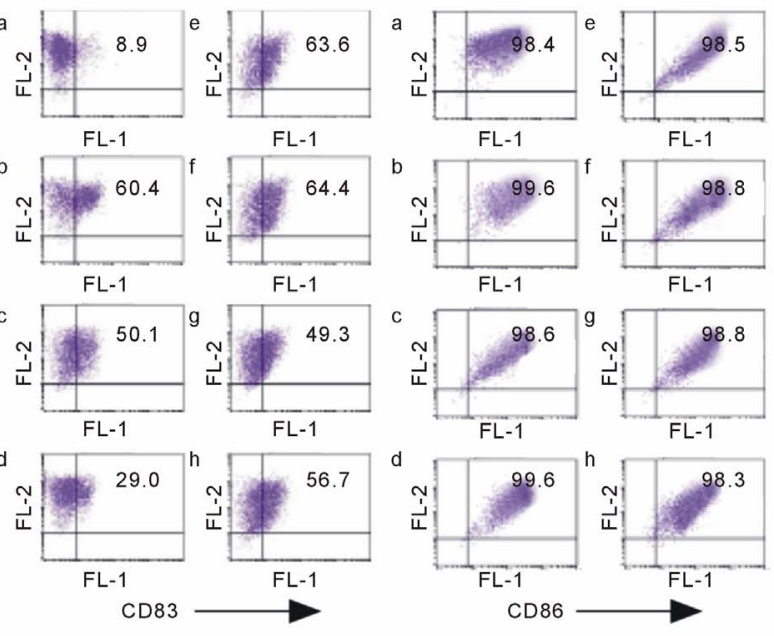

(B)

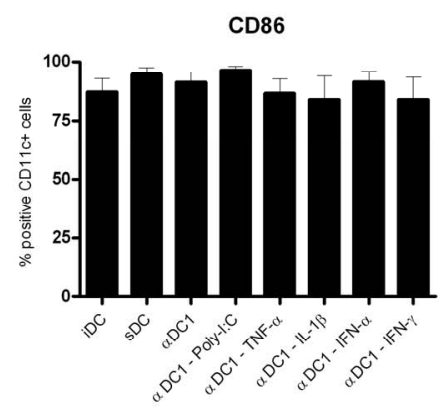

Figure 1. DC Phenotype. (A) DCs were initially identified as the large granular population based on flow cytometry FSC/SSC plots. Flow cytometric analysis was performed in order to determine the surface expression of the maturation markers CD80, CD83 and CD86 on CD11c + DCs; (B) The percentage of CD11c+ DCs staining positive of individual maturation markers in

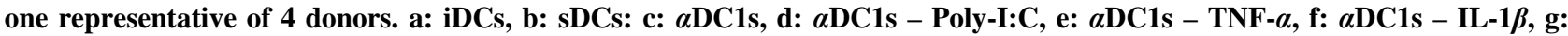
$\alpha \mathrm{DC1s}-\mathrm{IFN}-\alpha, \mathrm{h}: \alpha \mathrm{DC1s}-\mathrm{IFN}-\gamma$; (C) Mean percentage of maturation marker positive CD11c + DCs maturated according to the indicated cytokine cocktails $(n=4)$. $(*)$ indicates significant different maturation marker expression compared to iDCs $(p$ $<0.05)$, two-sided Students t-test.

of DCs maturated with all other $\alpha$-Type- 1 polarizing cocktail derivatives containing Poly-I:C was also reduced compared to sDCs and $\alpha \mathrm{DC} 1 \mathrm{~s}$ without Poly-I:C (Figure 2(B)). Exclusion of other cytokines from the $\alpha$-Type-1 polarizing cocktail did not significantly alter the percentage of viable DCs. However, inclusion of Poly-I:C in a maturation cocktail does not seem to reduce DC capacity for overall in-vitro allogeneic $\mathrm{T}$ cell stimulation (data not shown).

\subsection{Poly-I:C Mediated Effects on PKR Expression and Activation}

Reports of PKR activation upon direct binding of cytosolic Poly-I:C, and recruitment of PKR to a signaling complex binding to the cytosolic tail of TLR3 following Poly-I:C binding in the endosomal compartment, led to the speculation that the apparent Poly-I:C mediated reduction in DC viability might be caused by activation of
PKR. Here we demonstrate that PKR expression in sDCs was not elevated as compared to iDCs (Figures 3(A) and (B)). In contrast, PKR expression was elevated in $\alpha \mathrm{DC} 1 \mathrm{~s}$ as compared to iDCs and sDCs $(\mathrm{p}<0.05)$. However, no significant difference in PKR expression was found between DCs maturated using the $\alpha \mathrm{DC} 1$ cocktail with (14.7 fold increase) or without Poly-I:C (15.2 fold increase), suggesting that Poly-I:C has no effect on total DC PKR expression. In contrast, PKR expression of $\alpha \mathrm{DC} 1 \mathrm{~s}$ was dependent on IFN- $\alpha-(\mathrm{p}<0.05)$ (Figure 3(A) and (B)). Other subtractions did not lead to significant changes as compared to PKR expression in $\alpha \mathrm{DC} 1 \mathrm{~s}$.

As the role of Poly-I:C in PKR dynamics was expected to be found at the level of activation rather than on transcriptional regulation we next tested the levels of PKR Thr446 autophosphorylation in DCs and how absence of Poly-I:C affected PKR activation. Here, $\alpha \mathrm{DC} 1$ maturation resulted in autophosphorylation in 2 out of 4 donors, but we were unable to detect any differences in the levels 


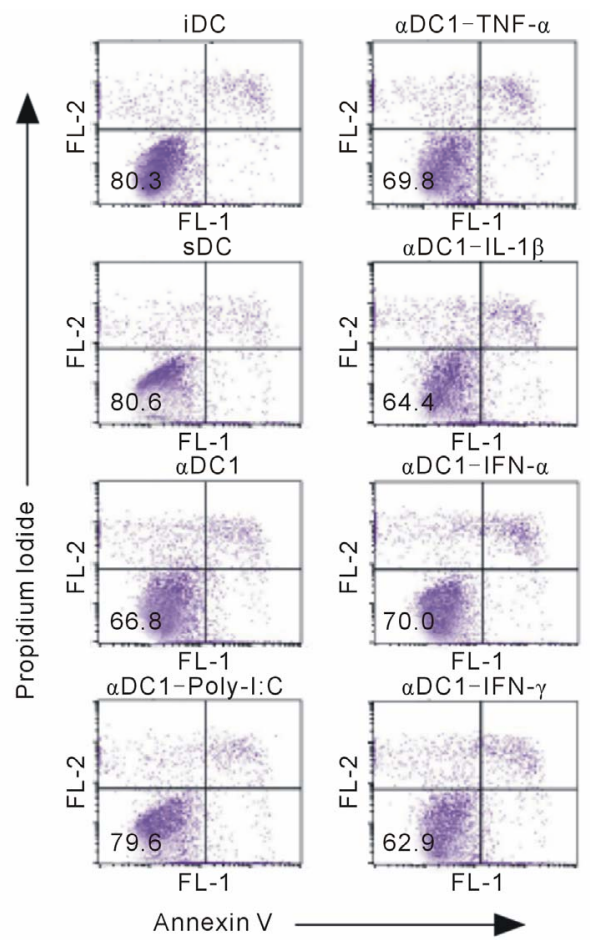

(A)

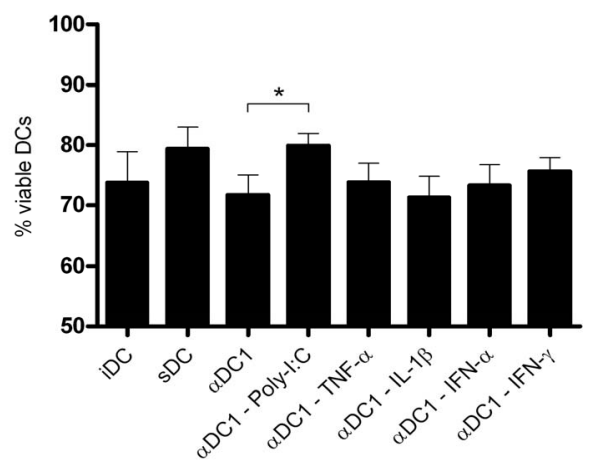

(B)

Figure 2. Exclusion of Poly-I:C from the $\alpha$-Type-1 polarizing cocktail increases $\alpha \mathrm{DC} 1$ viability. DCs maturated with the indicated cytokine cocktails were stained with annexin V FITC and PI, and subjected to flow cytometric analysis. (A) Dot plots of annexin V FITC and PI stained DCs from one representative of 8 donors. The percentage of viable cells is indicated in the lower left corner; (B) Mean percentage of viable cells from all donors $(n=8)$. $(*)$ indicates significant different levels of viability $(p<0.05)$, one-sided Students t-test.

of Thr446 phosphorylated PKR in $\alpha \mathrm{DC} 1 \mathrm{~s}$ and $\alpha \mathrm{DC} 1 \mathrm{~s}$ maturated without Poly-I:C for 24 or 48 hours (Figure 3(C) and data not shown) or during the first 6 hours after PolyI:C addition to DCs maturated with IFN- $\alpha$ (data not shown).

\section{Discussion}

Improving the quality of ex vivo generated DCs for

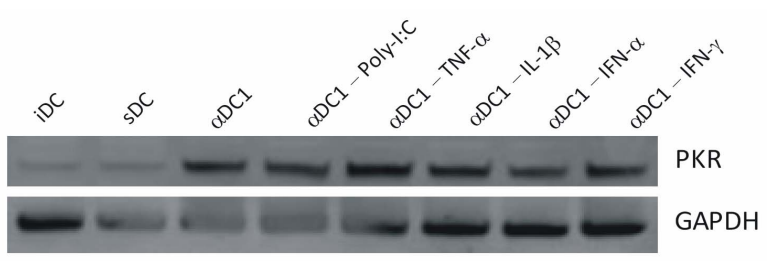

(A)

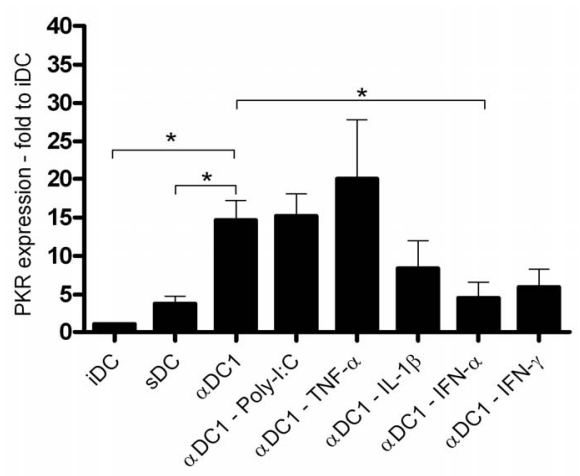

(B)

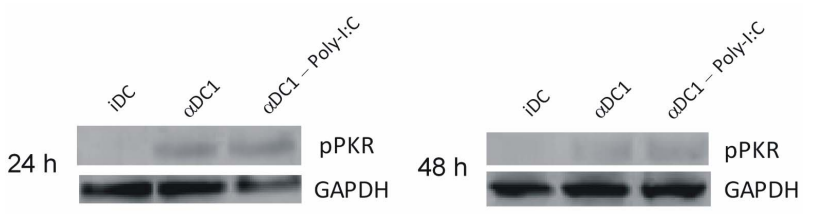

(C)

Figure 3. PKR expression and activation is unaffected by extracellular Poly-I:C. PKR expression was investigated by western blotting in DCs maturated with the indicated cytokine cocktails for 48 hours. (A) One representative blot is shown (n = 3); (B) PKR expression levels were normalized to GAPDH levels and the mean fold change in PKR expression compared to iDCs is plotted $(n=3)$. $(*)$ indicates significant different PKR expression ( $p<0.05$ ), two-sided Students t-test; (C) Active Thr446 phosphorylated PKR detection by western blotting was performed 24 and 48 hours after addition of the indicated maturation cocktails to iDCs. 2 out of 4 donors demonstrated Thr446 phosphorylated PKR. A western blot from one of these two donors is shown.

therapeutic cancer vaccination is of paramount importance and may increase the efficiency of this vaccine strategy. $\alpha \mathrm{DC} 1 \mathrm{~s}$ have been shown to be superior in IL-12p70 secretion and CTL activation [5]. Viability of therapeutic DCs is another factor of potential importance as DCs may exert their effect in vivo after more than 24 hours upon injection. In order to improve $\alpha \mathrm{DC} 1$ yields, we tried to identify the cause of Poly-I:C mediated DC death, in the attempt of identifying a target for future siRNA-mediated silencing strategies as a mean of generating functional $\alpha \mathrm{DC} 1 \mathrm{~s}$ with increased viability. In particular we investigated the potential role of PKR induction and autophosphorylation. 
Poly-I:C-mediated IL-12p70 secretion and induction of type I IFNs in $\alpha \mathrm{DC} 1 \mathrm{~s}$ [5] makes this TLR3 ligand indispensable in the $\alpha$-Type- 1 polarizing cocktail, and thus removal of Poly-I:C from the cytokine cocktail in order to restore DC viability is undesirable. However, apoptosis of DCs generated for therapeutic cancer vaccination is undesirable due to reduced DC yields from valuable and limited DC precursor cells and also decreases longevity upon in vivo injection. Furthermore, the presence of apoptotic cells can impair DC phenotypic maturation by suppressing the expression of co-stimulatory molecules [12], hereby impairing DC functionality and ultimately vaccine efficacy. However, this does not seem to occur at the percentage of dead cells occurring in our system (Figures 1(B) and (C)).

We hypothesized that activation of PKR in $\alpha \mathrm{DC} 1 \mathrm{~s}$ might cause the apparent Poly-I:C mediated cell death, as active PKR induces translational inhibition and apoptosis as a defense mechanism against viral infection $[7,8]$. Characterization of PKR expression in DCs revealed significantly higher amounts of PKR in $\alpha \mathrm{DC} 1 \mathrm{~s}$ and $\alpha \mathrm{DC} 1$ s maturated without Poly-I:C as compared to sDCs (Figure 3). The finding that presence of Poly-I:C has no effect on PKR expression is in compliance with the known role of Poly-I:C as a PKR activator and not as an inducer of PKR transcription [6]. The high PKR expression observed in $\alpha \mathrm{DC} 1 \mathrm{~s}$ seems to be induced by IFNs, as $\alpha \mathrm{DC} 1 \mathrm{~s}$ maturated without IFN- $\alpha$ showed a significantly reduced PKR expression as compared to $\alpha \mathrm{DC} 1 \mathrm{~s}$. Maturation of $\alpha \mathrm{DC} 1 \mathrm{~s}$ without IFN- $\gamma$ also seemed to reduce PKR expression as compared to $\alpha \mathrm{DC} 1 \mathrm{~s}$ however this reduced expression was non-significant. These results confirm the established role of IFNs as inducers of PKR expression (reviewed in [13]).

Despite high PKR expression levels in $\alpha \mathrm{DC} 1 \mathrm{~s}$, we observed no significant difference in the amounts of active Thr446 phosphorylated PKR in $\alpha \mathrm{DC} 1 \mathrm{~s}$ and $\alpha \mathrm{DC} 1 \mathrm{~s}$ maturated without Poly-I:C (Figure 3(C)). The observed lack of Poly-I:C mediated PKR activation at neither 48 hours after addition nor during the first 6 hours (data not shown) suggests that PKR is not activated by extracellular Poly-I:C. These results argue against a role of activated PKR as the inducer of cell death in $\alpha \mathrm{DC} 1 \mathrm{~s}$ despite our previous findings of Poly-I:C as the cause of the lowered viability of $\alpha \mathrm{DC} 1 \mathrm{~s}$. The apparent discrepancy regarding the ability of Poly-I:C to induce Thr446 PKR autophosphorylation in our hands and others might be due to Poly-I:C residing in different compartments. PKR activation by Poly-I:C reported by McAllister et al. was observed after transfection, hereby enabling direct cytosolic binding of Poly-I:C to PKR [6], whereas Poly-I:C was added extracellularly in our protocol for DC maturation. We were unable to find any reports of translocation of Poly-I:C from the endosomal compartment to the cy- toplasm of DCs, thus explaining the lack of PKR activation. Furthermore, PKR activation following recruitment to a TLR3 signaling complex in the endosome, activated upon Poly-I:C addition was not directly detected by Jiang et al., as the effect of transfection with dominant negative PKR was only investigated on the level of NF- $\mathrm{KB}$ activtion [10].

In conclusion, we identified a Poly-I:C dependent lowered viability of $\alpha \mathrm{DC} 1 \mathrm{~s}$ where the mechanisms of apoptosis was independent of PKR expression and activation. Future studies may reveal the targets for this Poly-I:C mediated apoptosis which could be exploited for siRNA silencing, thus maintaining the efficient $\alpha \mathrm{DC} 1$ mediated maturation without hampering DC viability as compared to sDCs.

\section{Acknowledgements}

This work was supported by the Faculty of Health Sciences, University of Copenhagen and a grant from The Augustinus Foundation.

\section{REFERENCES}

[1] F. Sallusto and A. Lanzavecchia, "Efficient Presentation of Soluble Antigen by Cultured Human Dendritic Cells Is Maintained by Granulocyte/Macrophage Colony-Stimulating Factor plus Interleukin 4 and Downregulated by Tumor Necrosis Factor Alpha," The Journal of Experimental Medicine, Vol. 179, No. 4, 1994, pp. 1109-1118. doi:10.1084/jem.179.4.1109

[2] I. J. de Vries, W. J. Lesterhuis, N. M. Scharenborg, L. P. Engelen, D. J. Ruiter, M. J. Gerritsen, et al., "Maturation of Dendritic Cells Is a Prerequisite for Inducing Immune Responses in Advanced Melanoma Patients," Clinical Cance Research, Vol. 9, 2003, p. 5091.

[3] H. Jonuleit, U. Kuhn, G. Muller, K. Steinbrink, L. Paragnik, E. Schmitt, et al., "Pro-Inflammatory Cytokines and Prostaglandins Induce Maturation of Potent Immunostimulatory Dendritic Cells under Fetal Calf Serum-Free Conditions," European Journal of Immunology, Vol. 27, No. 12, 1997, pp. 3135-3142.

doi:10.1002/eji.1830271209

[4] R. Trepiakas, A. E. Pedersen, O. Met, M. H. Hansen, A. Berntsen and I. M. Svane, "Comparison of Alpha-Type-1 Polarizing and Standard Dendritic Cell Cytokine Cocktail for Maturation of Therapeutic Monocyte-Derived Dendritic Cell Preparations from Cancer Patients," Vaccine, Vol. 26, No. 23, 2008, pp. 2824-2832.

doi:10.1016/j.vaccine.2008.03.054

[5] R. B. Mailliard, A. Wankowicz-Kalinska, Q. Cai, A. Wesa, C. M. Hilkens, M. L. Kapsenberg, et al., "AlphaType-1 Polarized Dendritic Cells: A Novel Immunization Tool with Optimized CTL-Inducing Activity," Cancer Research, Vol. 64, No. 17, 2004, pp. 5934-5937. doi:10.1158/0008-5472.CAN-04-1261

[6] C. S. McAllister and C. E. Samuel, "The RNA-Activated Protein Kinase Enhances the Induction of Interferon-Beta 
and Apoptosis Mediated by Cytoplasmic RNA Sensors," Journal of Biological Chemistry, Vol. 284, No. 3, 2009, pp. 1644-1651. doi:10.1074/jbc.M807888200

[7] F. Zhang, P. R. Romano, T. Nagamura-Inoue, B. Tian, T. E. Dever, M. B. Mathews, et al., "Binding of DoubleStranded RNA to Protein Kinase PKR Is Required for Dimerization and Promotes Critical Autophosphorylation Events in the Activation Loop," Journal of Biological Chemistry, Vol. 276, No. 27, 2001, pp. 24946-24958. doi:10.1074/jbc.M102108200

[8] D. Scheuner, R. Patel, F. Wang, K. Lee, K. Kumar, J. Wu, et al., "Double-Stranded RNA-Dependent Protein Kinase Phosphorylation of the Alpha-Subunit of Eukaryotic Translation Initiation Factor 2 Mediates Apoptosis," Journal of Biological Chemistry, Vol. 281, No. 30, 2006, pp. 21458-21468. doi:10.1074/jbc.M603784200

[9] L. Alexopoulou, A. C. Holt, R. Medzhitov and R. A. Flavell, "Recognition of Double-Stranded RNA and Activation of NF-kappa B by Toll-Like Receptor 3," Nature, Vol. 413, No. 6857, 2001, pp. 732-738. doi:10.1038/35099560

[10] Z. Jiang, M. Zamanian-Daryoush, H. Nie, A. M. Silva, B. R. Williams and X. Li, "Poly(I-C)-Induced Toll-Like
Receptor 3 (TLR3)-Mediated Activation of NFkappa B and MAP Kinase Is through an Interleukin-1 ReceptorAssociated Kinase (IRAK)-Independent Pathway Employing the Signaling Components TLR3-TRAF6-TAK1TAB2-PKR," Journal of Biological Chemistry, Vol. 278, No. 19, 2003, pp. 16713-16719. doi:10.1074/jbc.M300562200

[11] A. E. Pedersen, M. Thorn, M. Gad, M. R. Walter, H. E. Johnsen, E. Gaarsdal, et al., "Phenotypic and Functional Characterization of Clinical Grade Dendritic Cells Generated from Patients with Advanced Breast Cancer for Therapeutic Vaccination," Scandinavian Journal of Immunology, Vol. 61, No. 2, 2005, pp. 147-156. doi:10.1111/j.0300-9475.2005.01531.x

[12] C. A. Williams, R. A. Harry and J. D. McLeod, "Apoptotic Cells Induce Dendritic Cell-Mediated Suppression via Interferon-Gamma-Induced IDO," Immunology, Vol. 124, No. 1, 2008, pp. 89-101. doi:10.1111/j.1365-2567.2007.02743.x

[13] M. A. Garcia, E. F. Meurs and M. Esteban, "The dsRNA Protein Kinase PKR: Virus and Cell Control," Biochimie, Vol. 89, No. 6-7, 2007, pp. 799-811. doi:10.1016/j.biochi.2007.03.001 